\title{
Collisions for the Quantum Coulomb Hamiltonian
}

\author{
C. Gérard ${ }^{1}$ and A. Knauf ${ }^{2}$ \\ ${ }^{1}$ Centre de Mathématiques, Ecole Polytechnique, F-91128 Palaiseau, Cedex, France \\ 2 Technische Universität Fachbereich Mathematik, Straße des 17 Juni, 135, 1000 Berlin, \\ Federal Republic of Germany
}

Received February 26, 1991; in revised form April 16, 1991

\begin{abstract}
We study the propagation of phase space singularities for the time dependent Schrödinger equation with potential having Coulomb-type singularities in space dimension equal to three. We prove that the singularities (frequency set) of the solution are reflected by a Coulomb center exactly as in the classical problem, i.e. the frequency set follows the regularized trajectories of Classical Mechanics after a collision.
\end{abstract}

\section{Introduction}

In this paper we study the problem of diffraction of singularities by Coulomb centers for solutions of the time dependent Schrödinger equation:

$$
\left\{\begin{array}{l}
i h \partial_{t} u=H u \\
u_{\mid t=0}=u_{0}
\end{array} .\right.
$$

Here $H$ is a semiclassical Schrödinger operator of the form $H=-h^{2} \Delta+V(x)$, on $L^{2}\left(\mathbb{R}^{3}\right)$, where $V(x)$ is a real potential having Coulomb-type singularities at some points $x^{i}$ in $\mathbb{R}^{3}$.

We want here to understand an important difference between Classical and Quantum Mechanics for Coulomb potentials. Namely in Classical Mechanics, there exist collision trajectories, i.e. trajectories which hit a singularity of the potential in finite time. To extend the trajectory after the collision, one must use a procedure known as regularization. On the contrary, in Quantum Mechanics, the evolution is defined for all times for Coulomb potentials, which can be seen as a manifestation of the uncertainty principle. So it is interesting to study the semiclassical limit $h \rightarrow 0$ of the quantum evolution for times and initial conditions which would give rise to a collision for the classical problem. This is what we study in this paper.

The Frequency Set. Let us first precise what we mean by singularities of the 
solution $e^{-i t H / h} u_{0}$ of (1). We consider in this paper the notion of singularities in phase space which is natural in semiclassical problems, namely the notion of frequency set. Let us first recall the definition of frequency set denoted in this paper by $F S$ (see Guillemin-Sternberg [Gu-St]). We denote by $H^{k}(\Omega)$ the usual Sobolev space of order $k$ on some open set $\Omega$.

Definition 1.1. Let $u_{h} \in D^{\prime}\left(\mathbb{R}^{n}\right)$ be a $h$-dependent distribution such that for any bounded open set $\Omega \subset \mathbb{R}^{n}$ there exist some $N_{0} \in \mathbb{N}$ with

$$
\left\|u_{h}\right\|_{H^{-N_{0}(\Omega)}}=O\left(h^{-N_{0}}\right)
$$

Then a point $\left(x_{0}, \xi_{0}\right) \in T^{*}\left(\mathbb{R}^{n}\right)$ is not in $F S u_{h}$ if there exist a function $\chi(x, \xi) \in C_{0}^{\infty}\left(T^{*}\left(\mathbb{R}^{n}\right)\right)$ with $\chi\left(x_{0}, \xi_{0}\right) \neq 0$ and $\chi\left(x, h D_{x}\right) u_{h}=O\left(h^{\infty}\right)$ in $L^{2}\left(\mathbb{R}^{n}\right)$.

In the definition and in all the paper, $\chi\left(x, h D_{x}\right)$ will denote the Weyl quantization of the symbol $\chi(x, \xi)$, defined by:

$$
\chi\left(x, h D_{x}\right) u(x, h)=(2 \pi h)^{-n} \int e^{i(x-y) \xi / h} \chi\left(\frac{x+y}{2}, \xi\right) u(y, h) d y d \xi
$$

So the frequency set characterize the set of points in phase space where the distribution $u_{h}$ is strongly oscillating when $h$ tends to 0 .

If $H=-h^{2} \Delta+V(x)$ and $V(x)$ is a smooth potential it is well known (see for example Robert [Ro]) that with the notations of (1) one has:

$$
F S e^{-i t \boldsymbol{H} / \boldsymbol{h}} u_{0}=\phi_{t}\left(F S u_{0}\right)
$$

where $\phi_{t}$ is the Hamiltonian flow $\exp \left(t H_{p}\right)$, and $p=\xi^{2}+V(x)$.

The problem we address in this paper is to extend this result to potentials having Coulomb type singularities when the space dimension is equal to three. More precisely, we want to describe for example the frequency set of $u_{t}$ when $u_{0}$ has frequency set near a point $\left(x_{0}, \xi_{0}\right)$ such that the trajectory starting from this point arrives at a Coulomb center in a finite time (i.e. giving rise to a collision for the classical problem).

The Potential and the Regularized Trajectories. We will now precise the hypotheses on the potential $V$ and describe the regularized trajectories which will allow us to extend the result known for smooth potentials.

Hypotheses on the Potential. Our main interest in this paper will be the physically important example of the Coulomb potential with many centers:

$$
V(x)=\sum_{1}^{n} \frac{c_{i}}{\left|x-x^{i}\right|}+\tilde{V}(x)
$$

where $x^{1}, \ldots, x^{n}$ are points in $\mathbb{R}^{3}$, and $\tilde{V}$ is a smooth real bounded potential. Our approach will be to apply to the quantum problem a method of regularization used in the corresponding classical problem in Celestial Mechanics. This method, known as the Kustaanheimo-Stiefel transformation, will be introduced in Sect. 2. Even though this method was introduced in Celestial Mechanics to regularize collisions for Coulomb potentials, one can apply it to a larger class of potentials. We will prove our result (Theorem 1) only for Coulomb potentials, but the proofs 
extend directly to the class of potentials satisfying the following hypotheses:

(H1): $V(x)$ is real and smooth outside a finite set of points $x^{1}, \ldots, x^{n} \in \mathbb{R}^{3}$.

(H2): $V$ is $\Delta$ bounded with relative bound strictly less than 1 , so that $H$ is selfadjoint with domain $H^{2}\left(\mathbb{R}^{3}\right)$ (this is probably not really necessary but simplifies the problem).

We will now describe the type of singularities of $V$ allowed near a point $x^{i}$. Near a singular point $x^{i}$ for $1 \leqq i \leqq n$ (we may assume that $x^{i}=0$ ) we assume that the singularity of $V$ is of the following type: if $x=\left(x_{1}, x_{2}, x_{3}\right)$, we put: $x=\Lambda(z) z$, where:

$$
\Lambda(z)=\left(\begin{array}{cccc}
z_{1} & -z_{2} & -z_{3} & z_{4} \\
z_{2} & z_{1} & -z_{4} & -z_{3} \\
z_{3} & z_{4} & z_{1} & z_{2}
\end{array}\right) .
$$

Note that one has: $r=\left(x_{1}^{2}+x_{2}^{2}+x_{3}^{2}\right)^{1 / 2}=z_{1}^{2}+z_{2}^{2}+z_{3}^{2}+z_{4}^{2}$. Then we ask:

(H3): $W(z)=z^{2} V(\Lambda(z) z)$ is a smooth function of the variables $z \in \mathbb{R}^{4}$ such that $\lim _{z \rightarrow 0} W(z)=c_{i} \neq 0$.

For example we can replace in (2) the Coulomb potential by the Yukawa potential $|x|^{-1} e^{-\mu|x|}$ for $\mu>0$.

We will now define the regularized trajectories for this class of potentials. It clearly suffices to extend a trajectory of the Hamiltonian flow after a collision with a singular point $x^{i}$ which we suppose to be at the origin. The problem of regularizing collisions is a very old one in Celestial Mechanics (see for example Stiefel-Scheiffele [S-S], Saari [Sa]). We will follow the method of the Kustaanheimo-Stiefel transformation (see Stiefel-Scheiffele $[\mathrm{S}-\mathrm{S}]$ for more details).

To find the regularized trajectory starting from a point $\left(x_{0}, \xi_{0}\right)$, with $x_{0} \neq 0$, one first chooses a point $z_{0}$ such that $x_{0}=\Lambda\left(z_{0}\right) z_{0}$, and a point $\zeta_{0}$ such that $\xi_{0}=\left(2 z_{0}^{2}\right)^{-1} \Lambda\left(z_{0}\right) \zeta_{0}$. Such points are of course non-unique but the regularized flow does not depend on the choice of $\left(z_{0}, \zeta_{0}\right)$ (see $\left.[\mathrm{S}-\mathrm{S}]\right)$. Let us denote by $(x, \xi)=\Lambda^{*}(z, \zeta)$ this transformation. One then denotes by $(z(s), t(s), \zeta(s), \tau(s))$ the trajectory in $T^{*}\left(\mathbb{R}_{z}^{4} \times \mathbb{R}_{t}\right)$ for the Hamiltonian $\tilde{p}=z^{2} \tau-\zeta^{2}-W(z)$ starting from the point $\left(z_{0}, 0, \zeta_{0}, \tau_{0}\right)$, where $z_{0}^{2} \tau_{0}-\zeta_{0}^{2}-W\left(z_{0}\right)=0$. Then we define:

Definition 1.2. The regularized flow denoted by $\tilde{\phi}_{t}: R_{t} \times T^{*}\left(R^{3} \backslash\{0\}\right) \rightarrow T^{*}\left(R^{3} \backslash\{0\}\right)$ is the mapping:

$$
\tilde{\phi}_{t}\left(x_{0}, \xi_{0}\right)=\Lambda^{*}\left(z_{s}, \zeta_{s}\right)
$$

where $s$ satisfies $t(s)=t$.

Remark 1. This definition can be understood more simply if one thinks of the transformation $x \rightarrow z$ as a kind of blow-up around the singularity. Then the change of parametrization of the Hamilton orbits due to the term $z^{2} \tau$ in $\tilde{p}$ amounts to introduce a slow time near the collision for which the velocity will stay bounded. For a purely Coulomb potential, the regularized trajectory is obtained by following backwards the incoming collision trajectory.

Remark 2. It is easy to see that outside the collision, the regularized flow is the same as the usual one. One can also introduce the regularized flow in a more 
topological way by introducing a new phase space obtained by blowing up each singularity of the potential and by extending suitably the flow at the singularity (see for example Moser [Mo], Easton [Ea]).

Results. We now state the results of this paper. For a given open set $\Omega \subset T^{*}\left(\mathbb{R}^{3} \backslash\{0\}\right)$, and for $t \in \mathbb{R}$, we denote by $\operatorname{Coll}_{t}(\Omega) \subset \Omega$ the set of points $(x, \xi) \in \Omega$ such that there exists $i$ with $\pi_{x} \tilde{\phi}_{s}(x, \xi) \rightarrow x^{i}$ when $s \rightarrow t^{-}$. So $\operatorname{Coll}_{t}(\Omega)$ is the set of initial conditions in $\Omega$ which will give rise to a collision for the classical flow, (extended by regularization across possible previous collisions). Then we have the following theorem:

Theorem 1. Let $u_{0} \in L^{2}\left(\mathbb{R}^{3}\right)$ such that:

i) there exists some $N_{0} \in \mathbb{N}$ with:

$$
\left\|u_{0}\right\|_{L^{2}}=O\left(h^{-N_{0}}\right)
$$

ii) there exists a cutoff function $\chi_{0}(x, \xi) \in C_{0}^{\infty}$ supported away from $\left\{x^{1}, \ldots, x^{n}\right\} \times \mathbb{R}^{3}$ with:

$$
u_{0}=\chi_{0}\left(x, h D_{x}\right) u_{0}+O_{H^{2}}\left(h^{\infty}\right) .
$$

Here $\mathrm{O}_{\mathrm{H}^{2}}\left(h^{\infty}\right)$ means that the remainder term has a $\mathrm{H}^{2}$ norm of size $O\left(h^{\infty}\right)$. Then one has:

$$
F S e^{-i t H / h} u_{0} \cap T^{*}\left(\mathbb{R}^{3} \backslash\left\{x^{1}, \ldots, x^{n}\right\}\right)=\tilde{\phi}_{t}\left(F S u_{0} \backslash \operatorname{Coll}_{t}\left(F S u_{0}\right)\right),
$$

2) if $\operatorname{Coll}_{t}\left(F S u_{0}\right)=\varnothing$ then:

$$
F S e^{-i t H / h} u_{0}=\tilde{\phi}_{t}\left(F S u_{0}\right)
$$

To illustrate this result, let us for example consider what happens when $F S u_{0}=\left(x_{0}, \xi_{0}\right)$ and when for simplicity the only Coulomb center is at 0 . Then if the trajectory $\phi_{t}\left(x_{0}, \xi_{0}\right)$ never reaches 0 , the frequency set of $e^{-i t H / h} u_{0}$ is equal to $\phi_{t}\left(x_{0}, \xi_{0}\right)$ as for smooth potentials. If the trajectory $\phi_{t}\left(x_{0}, \xi_{0}\right)$ hits 0 at time $T_{c}$, then for $t \neq T_{c}$, the frequency set of $e^{-i t H / h} u_{0}$ is equal to $\tilde{\phi}_{t}\left(x_{0}, \xi_{0}\right)$. For $t=T_{c}$, one just knows that the frequency set of $e^{-i T_{c} H / h} u_{0}$ is concentrated above 0 .

Remark 3. It seems quite likely that the correct notion needed to study the singularity of $e^{-i T_{c} H / h} u_{0}$ is a version of the second microlocalization above 0 which is adapted to the Kustaanheimo-Stiefel transformation.

Remark 4. Using the arguments of the proof of Lemma 3.1, we easily see that if $u_{0}=\chi(H) \chi_{0}(x) u_{1}$, for $u_{1} \in L^{2}\left(\mathbb{R}^{3}\right), \chi_{0}(x) \in C_{0}^{\infty}\left(\mathbb{R}^{3} \backslash\left\{x^{1}, \ldots, x^{n}\right\}\right)$ and $\chi(\lambda) \in C_{0}^{\infty}(\mathbb{R})$, then there exist some cutoff function $\chi_{0}(x, \xi)$ supported away from $\left\{x^{1}, \ldots, x^{n}\right\} \times \mathbb{R}^{3}$ such that:

$$
\chi_{0}\left(x, h D_{x}\right) u_{0}=u_{0}+O_{H^{2}}\left(h^{\infty}\right)
$$

so one can apply Theorem 1 to $u_{0}$.

Remark 5. It is interesting to see if the results of Theorem 1 extend to the case of analytic frequency set (see Martinez [Ma] for a definition), where roughly $O\left(h^{\infty}\right)$ estimates are replaced by $O\left(e^{-c / h}\right)$ estimates. For example in the case of scattering by an obstacle, it is known that $C^{\infty}$ and analytic singularities propagate differently (see for Sjöstrand [Sj], Lebeau [Le]). If the transformed potential $W$ is analytic then it is straightforward to see that part 1) of Theorem 1 extends to analytic 
frequency set. To extend part 2) one would probably need to construct a version of the functional calculus of Helffer-Robert [He-Ro] adapted to analytic theory.

Let us give now the plan of this paper. In Sect. 2, we quantize the Kustaanheimo Stiefel transformation to reduce the time dependent Schrödinger equation to an equation with smooth coefficients. In Sect. 3, we prove Theorem 1.

\section{The KS Transform}

In this section we will quantize the Kustaanheimo-Stiefel transform to reduce the time dependent Schrödinger equation to an equation with smooth coefficients. Recall that the KS transformation: $z \in \mathbb{R}^{4} \rightarrow x \in \mathbb{R}^{3}$ is given by $x=\Lambda(z) z$, where:

$$
\Lambda(z)=\left(\begin{array}{cccc}
z_{1} & -z_{2} & -z_{3} & z_{4} \\
z_{2} & z_{1} & -z_{4} & -z_{3} \\
z_{3} & z_{4} & z_{1} & z_{2}
\end{array}\right) .
$$

We begin by finding local inverses to the KS transformation if we add to $x \in \mathbb{R}^{3}$ a dummy variable $\theta \in S^{1}$. Over $x \neq 0, x_{1}>0$, we denote by $\chi_{+}: \mathbb{R}^{3} \times S^{1} \rightarrow \mathbb{R}^{4}$ the following map:

$$
\begin{aligned}
& z_{1}=\sqrt{2}^{-1}\left(x_{1}+r\right)^{1 / 2} \cos \theta, \\
& z_{2}=\sqrt{2}^{-1}\left(x_{1}+r\right)^{-1 / 2}\left(x_{2} \cos \theta+x_{3} \sin \theta\right), \\
& z_{3}=\sqrt{2}^{-1}\left(x_{1}+r\right)^{-i / 2}\left(x_{3} \cos \theta-x_{2} \sin \theta\right), \\
& z_{4}=\sqrt{2}^{-1}\left(x_{1}+r\right)^{1 / 2} \sin \theta .
\end{aligned}
$$

Over $x \neq 0, x_{1}<0$, we denote by $\chi_{-}: \mathbb{R}^{3} \times S^{1} \rightarrow \mathbb{R}^{4}$ the following map:

$$
\begin{aligned}
& z_{2}=\sqrt{2}^{-1}\left(-x_{1}+r\right)^{1 / 2} \cos \theta, \\
& z_{1}=\sqrt{2}^{-1}\left(-x_{1}+r\right)^{-1 / 2}\left(x_{2} \cos \theta+x_{3} \sin \theta\right), \\
& z_{4}=\sqrt{2}^{-1}\left(-x_{1}+r\right)^{-1 / 2}\left(x_{3} \cos \theta-x_{2} \sin \theta\right), \\
& z_{3}=\sqrt{2}^{-1}\left(-x_{1}+r\right)^{1 / 2} \sin \theta .
\end{aligned}
$$

Here $r=\left(x_{1}^{2}+x_{2}^{2}+x_{3}^{2}\right)^{1 / 2}=z_{1}^{2}+z_{2}^{2}+z_{3}^{2}+z_{4}^{2}$. It is a straightforward computation to check that:

$$
d z_{1} d z_{2} d z_{3} d z_{4}=C_{0} r^{-1} d x_{1} d x_{2} d x_{3} d \theta \text {. }
$$

Then if $x \neq 0, \pm x_{1}>0$ and $z=\chi_{ \pm}(x, \theta)$, one has: $x=\Lambda(z) z, e^{i \theta}=2^{1 / 2}\left(x_{1}+r\right)^{-1 / 2}\left(z_{1}+i z_{4}\right)$ in the + case and $e^{i \theta}=2^{1 / 2}\left(-x_{1}+r\right)^{-1 / 2}\left(z_{2}+i z_{3}\right)$ in the - case. One also notices that in $|x| \geqq \varepsilon, \chi_{ \pm}$is actually an inverse to the KS transformation not only in $\pm x_{1}>0$, but in $\pm x_{1}>-\varepsilon$.

We consider now the Hamiltonian $H=-h^{2} \Delta_{x}-|x|^{-1}+V(x)$ on $L^{2}\left(\mathbb{R}^{3}\right)$, where $V$ is a real smooth bounded potential. $H$ is selfadjoint with domain $H^{2}\left(\mathbb{R}^{3}\right)$, and for an initial data $u_{0} \in H^{2}\left(\mathbb{R}^{3}\right)$, we denote by $u(x, t)$ the function $e^{-i t H / h} u_{0}$.

Then $u$ belongs to $C^{0}\left(\mathbb{R}_{t}, H^{2}\left(\mathbb{R}_{x}^{3}\right)\right) \cap C^{1}\left(\mathbb{R}_{t}, L^{2}\left(\mathbb{R}_{x}^{3}\right)\right)$, and since $H^{2}\left(\mathbb{R}^{3}\right) \subset C^{0}\left(\mathbb{R}^{3}\right)$, we can define the transformed function $\tilde{u}(z, t)$ by:

$$
\tilde{u}(z, t)=K u(z, t)=u\left(z_{1}^{2}-z_{2}^{2}-z_{3}^{2}+z_{4}^{2}, 2\left(z_{1} z_{2}-z_{3} z_{4}\right), 2\left(z_{1} z_{3}+z_{2} z_{4}\right), t\right) .
$$


We will prove the following proposition:

Proposition 2.1. The function $\tilde{u}$ satisfies the following equation in $D^{\prime}\left(\mathbb{R}_{t} \times \mathbb{R}_{z}^{4}\right)$ :

$$
i h z^{2} \partial_{t} \tilde{u}=-h^{2} \Delta_{z} \tilde{u}-\tilde{u}+V_{1}(z) \tilde{u} .
$$

Here $V_{1}(z)=z^{2} V(\Lambda(z))$.

Proof. We begin by transforming the equation satisfied by $u$. Since $C_{0}^{\infty}$ is dense in the domain of $H$, we know that $u$ satisfies the Schrödinger equation:

$$
i h \partial_{t} u=-h^{2} \Delta_{x} u-\frac{1}{|x|} u+V(x) u
$$

in distribution sense on $\mathbb{R}_{t} \times \mathbb{R}_{x}^{3}$. Indeed $|x|^{-1} \in L^{2}\left(\mathbb{R}^{3}\right)+L^{\infty}\left(\mathbb{R}^{3}\right)$, so each term in (4) is in $C^{0}\left(\mathbb{R}_{t}, L^{2}\left(\mathbb{R}_{x}^{3}\right)\right)$. From this it follows also that $u$ satisfies:

$$
i h|x| \partial_{t} u=-h^{2}|x| \Delta_{x} u-u+|x| V(x) u \text { in } D^{\prime}\left(\mathbb{R}_{t} \times \mathbb{R}_{x}^{3}\right) .
$$

Let us now take a sequence of smooth functions $u_{n}$ such that:

$$
u_{n} \rightarrow u \text { in } C^{0}\left(\mathbb{R}_{t}, H^{2}\left(\mathbb{R}_{x}^{3}\right)\right) \text {. }
$$

By a direct computation we see that:

$$
\Delta_{z} K u_{n}=|z|^{2} K \Delta_{x} u_{n} .
$$

Since $K u_{n} \rightarrow K u$ in $C^{0}\left(\mathbb{R}_{t}, C^{0}\left(\mathbb{R}_{z}^{4}\right)\right)$, we know that $\Delta_{z} K u_{n} \rightarrow \Delta_{z} K u$ in $C^{0}\left(\mathbb{R}_{t}, D^{\prime}\left(\mathbb{R}_{z}^{4}\right)\right)$. We also have that $\Delta_{x} u_{n} \rightarrow \Delta_{x} u$ in $C^{0}\left(\mathbb{R}_{t}, L^{2}\left(\mathbb{R}_{x}^{3}\right)\right)$. We now consider $u(x, t)$ as a function depending also on the dummy variable $\theta \in S^{1}$. Using (3), it is easy to get that $K \Delta_{x} u$ and $K \Delta_{x} u_{n}$ are in $C^{0}\left(\mathbb{R}_{t}, L^{2}\left(\mathbb{R}_{z}^{4},|z|^{2} d z\right)\right.$ ), and that:

$$
K \Delta_{x} u_{n} \rightarrow K \Delta_{x} u \text { in } C^{0}\left(\mathbb{R}_{t}, L^{2}\left(\mathbb{R}_{z}^{4},|z|^{2} d z\right)\right) \text {. }
$$

So we get that:

$$
|z|^{2} K \Delta_{x} u_{n} \rightarrow|z|^{2} K \Delta_{x} u \quad \text { in } C^{0}\left(\mathbb{R}_{t}, L_{\text {loc }}^{2}\left(\mathbb{R}_{z}^{4}, d z\right)\right) .
$$

This proves that $\Delta_{z} K u=|z|^{2} K \Delta_{x} u$. By the same argument we get that since $\partial_{t} u \in C^{0}\left(\mathbb{R}_{t}, L^{2}\left(\mathbb{R}_{x}^{3}\right)\right),|z|^{2} \partial_{t} \tilde{u} \in C^{0}\left(\mathbb{R}_{t}, L^{2}\left(\mathbb{R}_{z}^{4}\right)\right)$. The same is true from $\tilde{u}$ and $|z|^{2} V(\Lambda(z)(z)) \tilde{u}$. So this shows that $\tilde{u}$ satisfies the proposition.

\section{Proof of the Theorem}

In this section we will prove Theorem 1 . We begin by a few remarks. Recall from the Introduction that we consider an initial data $u_{0}(x, h)$ (the $h$ dependence of $u$ and $u_{0}$ will often be omitted to simplify notations) such that $\left\|u_{0}\right\|_{L^{2}}=O(h)^{-N_{0}}$ for some $N_{0}$. We assume that if $\chi_{0}(x, \xi)$ is a cutoff function supported away from $\left\{x^{1}, \ldots, x^{n}\right\} \times \mathbb{R}^{3}$, we have:

$$
u_{0}=\chi_{0}\left(x, h D_{x}\right) u_{0}+O_{H^{2}}\left(h^{\infty}\right) .
$$

Let now $\chi(\lambda)$ be a smooth cutoff function such that $\chi\left(\xi^{2}+V(x)\right)=1$ for $(x, \xi) \in \operatorname{supp} \chi_{0}$. Then we have the following result:

\section{Lemma 3.1.}

$$
\chi(H) u_{0}=u_{0}+O_{H^{2}}\left(h^{\infty}\right) .
$$


Proof. We begin by taking a smooth potential $\tilde{V}$ such that $\tilde{V}=V(x)$ on support of $\chi_{0}(x, \xi)$. If $\tilde{H}=-h^{2} \Delta_{x}+\tilde{V}$, it is straightforward using the functional calculus of Helffer-Robert [He-Ro] to get that:

$$
\chi(\tilde{H}) u_{0}=u_{0}+O_{H^{2}}\left(h^{\infty}\right) .
$$

It remains to check that $(\chi(\tilde{H})-\chi(H)) \chi_{1}(x)=O_{H^{2}}\left(h^{\infty}\right)$, if $\tilde{V}=V(x)$ on support of $\chi_{1}(x)$. To do this we use a formula due to Helffer-Sjöstrand [He-Sj] to represent $\chi(H)$. One has:

$$
\chi(H)=\frac{i}{2 \pi} \int_{\mathbb{C}} \frac{\partial \tilde{\chi}(z)}{\partial \bar{z}}(z-H)^{-1} d z \wedge d \bar{z} .
$$

Here $\tilde{\chi}(z)$ is an almost analytic extension of $\chi(\lambda)$ with compact support in $\mathbb{C}$, which satisfies $\partial_{\bar{z}} \tilde{\chi}(z)=O\left(|\operatorname{Im} z|^{\infty}\right)$, so the integral converges in operator norm. This formula gives:

$$
(\chi(H)-\chi(\tilde{H})) \chi_{1}(x)=\frac{i}{2 \pi} \int_{\mathbb{C}} \frac{\partial \tilde{\chi}(z)}{\partial \bar{z}}\left((z-H)^{-1}-(z-\tilde{H})^{-1}\right) \chi_{1} d z \wedge d \bar{z} .
$$

Then we have:

$$
\begin{aligned}
\left((z-H)^{-1}-(z-\tilde{H})^{-1}\right) \chi_{1} & =(z-H)^{-1}(H-\tilde{H})(z-\tilde{H})^{-1} \chi_{1} \\
& =(z-H)^{-1} \tilde{W}(z-\tilde{H})^{-1} \chi_{1},
\end{aligned}
$$

where $\tilde{W}=\tilde{V}-V(x)$ is supported where $\chi_{1}=0$. Then we have:

$$
\tilde{W}(z-\tilde{H})^{-1} \chi_{1}=\tilde{W}(z-\tilde{H})^{-1}\left[\tilde{H}, \chi_{1}\right](z-\tilde{H})^{-1}=O\left(\frac{h}{(\operatorname{Im} z)^{2}}\right) .
$$

This can be iterated since $\left[\tilde{H}, \chi_{1}\right]=\chi_{2}(x)\left[\tilde{H}, \chi_{1}\right]$, where $\chi_{2}$ is another cutoff with $\tilde{W} \chi_{2}=0$. This way we get finally a term in the integrand of (5) of norm between $L^{2}$ and $H^{2}$ equal to $O\left(h^{N}(\operatorname{Im} z)^{-N}\right)$ for any $N$, which completes the proof of the lemma since $\partial_{\bar{z}} \tilde{\chi}(z)=O(|\operatorname{Im} z|)^{\infty}$.

Before starting the proof of the theorem we make a few remarks. We will denote the function $e^{-i t H / h} u_{0}$ by $u$ if we consider it as a function of $(x, t)$ and by $u_{t}$ if we consider it as a function of $x$ alone. In Sect. 2 we introduced the transforms $\chi_{ \pm}$from $\left\{(x, \theta) \mid x \neq 0, \pm x_{1}>0\right\}$ into $\{z \mid z \neq 0\}$. We have seen that if we consider $u$ as a function of $(x, \theta, t)$ (which is of course independent of $\theta$ ), the transformed function $\tilde{u}(z)$ defined in Sect. 2 can also be viewed as the function $\tilde{u}=\chi_{ \pm}^{*} u$. We have the following lemma:

Lemma 3.2. For $x \neq 0$, one has:

$$
(x, t, \xi, \tau) \in F S u \Leftrightarrow(z, t, \zeta, \tau) \in F S \tilde{u},
$$

where:

$$
x=\Lambda(z) z, \quad \xi=\left(2 z^{2}\right)^{-1} \Lambda(z) \zeta .
$$

Proof. We will for the moment consider $u$ as a function depending also on the variable $\theta$. Since $\partial_{\theta} u=0$, we get:

$$
F S u(x, \theta, t)=\{(x, \theta, t, \xi, 0, \tau) \mid(x, t, \xi, \tau) \in F S u(x, t)\} .
$$


Then above $\mathbb{R}^{3} \backslash\{0\}$, we can apply the well known transformation rule of frequency set by the diffeomorphisms $\chi_{ \pm}$(see for example [Ro]) to get the result of the lemma. The fact that one does not need to compute $\theta=\theta(z)$ comes from the fact that the conjugate variable to $\theta$ is always 0 in (6).

Proof of Theorem 1. We will assume that the potential is a sum of Coulomb potentials as in (2), but the proof extends immediately to potentials satisfying hypotheses $(\mathrm{H})$. We will first consider the scattering of singularities by a single center, which we may assume to be the origin. We first consider the case of an attracting potential $(c<0)$. We will put $W(z)=c+V_{1}(z)$, where $V_{1}(0)=0$. Without loss of generality we can assume that $c=-1$.

The first step is to compute FSũ near $t=0$. Let us denote by $\Lambda_{*}:(z, \zeta) \rightarrow(x, \xi)$ the map defined in Lemma 3.2. We know from Lemma 3.2 that:

$$
F S \tilde{u} \cap\{z \neq 0\}=\left\{(z, t, \zeta, \tau) \mid\left(\Lambda^{*}(z, \zeta), t, \tau\right) \in F S u\right\} .
$$

(To avoid to burden the notations, we will sometimes allow ourselves to denote points in $T^{*}\left(\mathbb{R}_{x}^{3} \times \mathbb{R}_{t}\right)$ by $(x, \xi, t, \tau)$ instead of $(x, t, \xi, \tau)$.)

Since $u$ satisfies the equation:

$$
i h \partial_{t} u=-h^{2} \Delta_{x} u+V(x) u
$$

which has smooth coefficients above $x \neq 0$, we know that for $x \neq 0$, one has:

$$
(x, t, \xi, \tau) \in F S u \Leftrightarrow(x, \xi) \in F S u_{t}, \quad \tau=\xi^{2}+V(x) .
$$

To completely determine $F S \tilde{u}$, it remains to show that $\forall\left(\zeta_{0}, \tau_{0}\right),\left(0,0, \zeta_{0}, \tau_{0}\right) \notin F S \tilde{u}$. To do this we use that $\tilde{u}$ satisfies:

$$
-i h z^{2} \partial_{t} \tilde{u}=-h^{2} \Delta_{z} \tilde{u}-\tilde{u}+V_{1}(z) \tilde{u} .
$$

This implies that if $\left(0,0, \zeta_{0}, \tau_{0}\right) \in F S \tilde{u}$, then $\left|\zeta_{0}\right|=1$. If we consider now the Hamilton orbit for $\tilde{p}=z^{2} \tau-\zeta^{2}+1-V_{1}(z)$ starting from $\left(0,0, \zeta_{0}, \tau_{0}\right)$, we see that if $\left(0,0, \zeta_{0}, \tau_{0}\right) \in F S \tilde{u}$, then there would exist a point $\left(z_{1}, t_{1}, \zeta_{1}, \tau_{1}\right) \in F S \tilde{u}$ with $z_{1} \neq 0, z_{1}$ and $t_{1}$ arbitrarily small. Since over $z_{1} \neq 0$, we can apply Lemma 3.2, there would exist a point $\left(x_{1}, t_{1}, \xi_{1}, \tau_{1}\right) \in F S u$ with $x_{1} \neq 0, x_{1}$ and $t_{1}$ arbitrarily small.

On the other hand we can apply the standard result of propagation of frequency set for $u$, for small times and above $\mathbb{R}^{3} \backslash\{0\}$. This gives that for $t$ small, one has:

$$
F S u_{t} \cap\left(\mathbb{R}^{3} \backslash\{0\} \times \mathbb{R}^{3}\right)=\phi_{t}\left(F S u_{0}\right) .
$$

But clearly this cannot be very close to 0 for small times since $0 \notin \pi_{x}\left(F S u_{0}\right)$.

So we know that for $\varepsilon$ small enough, one has:

$$
F S \tilde{u} \cap\{-\varepsilon<t<\varepsilon\}=\left\{(z, t, \zeta, \tau) \mid\left(\Lambda^{*}(z, \zeta), t, \tau\right) \in F S u,-\varepsilon<t<\varepsilon\right\} .
$$

The proof of Theorem 1 will now consist in applying propagation of frequency set on $\tilde{u}$ and translate the results back to $u$ by Lemma 3.2. However Lemma 3.2 cannot be applied above 0 , so we first give a separate argument to describe what happens near $z=0$.

By Lemma 3.1, we can as well assume that:

$$
\chi(H) u_{0}=u_{0} .
$$

Indeed $e^{-i t H / h} u_{0}=e^{-i t H / h} \chi(H) u_{0}+O_{H^{2}}\left(h^{\infty}\right)$, so we get that $F S e^{-i t H / h} u_{0}=$ 
$F S e^{-i t H / h} \chi(H) u_{0}$. By the spectral theorem, we deduce from (10) that:

$$
\chi\left(-h D_{t}\right) u=-u \text {. }
$$

Since the transform $u \rightarrow \tilde{u}$ does not affect the $t$ variable, we also get:

$$
\chi\left(-h D_{t}\right) \tilde{u}=-\tilde{u} .
$$

So we can rewrite (8) as:

$$
\tilde{P} \tilde{u}=-i h z^{2} \chi\left(-h D_{t}\right) \partial_{t} \tilde{u}+h^{2} \Delta_{z} \tilde{u}+\tilde{u}-V_{1}(z) \tilde{u}=0 .
$$

Since for $|z|<C_{0},-z^{2} \tau \chi(-\tau)-\zeta^{2}+1-V(z)$ is elliptic for $|\zeta|>C_{1}$, for $C_{1}$ big enough, we can construct a pseudodifferential operator $Q\left(z, t, h D_{z}, h D_{t}, h\right)$ with principal symbol in $S\left(\langle\zeta\rangle^{2},\langle\zeta\rangle^{-2}\left(d \tau^{2}+d \zeta^{2}\right)\right)$ such that:

$$
K_{1}\left(z, t, h D_{z}, h D_{t}\right)=Q \tilde{P}+R_{\infty},
$$

where $R_{\infty}$ has a norm $O\left(h^{\infty}\right)$, and $K_{1}(z, t, \zeta, \tau)$ is a smooth bounded function supported in $\left\{(z, t, \zeta, \tau)|| z\left|\leqq C_{0}+1,\right| \zeta \mid \geqq C_{1}-1\right\}$ and equal to 1 in $\left\{(z, t, \zeta, \tau)|| z \mid \leqq C_{0}\right.$, $\left.|\zeta| \geqq C_{1}\right\}$.

We recall that a function $a(z, \zeta)$ belongs to the symbol class $S\left(\langle\zeta\rangle^{2}\right.$, $\left.\langle\zeta\rangle^{-2}\left(d \tau^{2}+d \zeta^{2}\right)\right)$ if:

$$
\left\|\partial_{z, t}^{\alpha} \partial_{\zeta, \tau}^{\beta} a\right\| \leqq C_{\alpha \beta}\langle\zeta\rangle^{2-|\beta|} .
$$

This and (11) give that if $K_{2}(z, t, \zeta, \tau)$ is supported in $\left\{(z, t, \zeta, \tau)|| z\left|\leqq C_{0},\right| \zeta \mid \geqq C_{1}\right.$, $\left.|\tau| \geqq C_{1}\right\}$, then one has:

$$
K_{2}\left(z, t, h D_{z}, h D_{t}\right) \tilde{u}=O_{L^{2}\left(\mathbb{R}_{z}^{4} \times \mathbb{R}_{t}\right)}\left(h^{\infty}\right) .
$$

Actually because of (11), $K_{2} \tilde{u}$ is also $O\left(h^{\infty}\right)$ in the norm $C^{0}\left(\mathbb{R}_{t}, L^{2}\left(\mathbb{R}_{z}^{4}\right)\right)$. We will use this remark later in the proof.

By the result of propagation of frequency set for Eq. (8), we know that $F S \tilde{u}$ is the union of Hamiltonian curves for $\tilde{p}$ starting from the points of $\left\{(z, t, \zeta, \tau) \mid\left(\Lambda^{*}(z, \zeta), t, \tau\right) \in F S u,-\varepsilon<t<\varepsilon\right\}$.

Let us first prove 1). The regularized trajectories are just the images under $\Lambda^{*}$ of the Hamiltonian curves for $\tilde{p}$ (see Definition 1.2). Then above $x \neq 0$, we can apply Lemma 3.2 and the argument leading to (7) to get that:

$$
F S u_{t} \cap\{x \neq 0\}=\tilde{\phi}_{t}\left(F S u_{0} \backslash \operatorname{Coll}_{t}\left(F S u_{0}\right)\right) .
$$

This proves 1).

To prove 2) it remains to prove that $u_{t}$ has no frequency set above $x=0$. By the definition of regularized trajectories, we know that $\tilde{u}$ has no frequency set above $\left(0, t_{0}\right)$, so an application of (13) gives that $\chi_{0}(t, z) \tilde{u}$ is $O\left(h^{\infty}\right)$ in $C^{0}\left(\mathbb{R}_{t}, L^{2}\left(\mathbf{R}_{z}^{4}\right)\right)$, if $\chi_{0} \in C_{0}^{\infty}$ is supported near $\left(0, t_{0}\right)$. This implies by the arguments of Sect. 2 that also $\chi_{0}(x, t) u$ is $O\left(h^{\infty}\right)$ in $C^{0}\left(\mathbb{R}_{t}, L^{2}\left(\mathbb{R}_{x}^{3}\right)\right)$. We use here that $u$ is independent of the variable $\theta$. This implies of course that $u_{t}$ has no frequency set above $x=0$. So this completes the proof of Theorem 1 in the attractive case.

Let us now consider the case of a repulsive potential, i.e. the case when $c>0$. Then of course no collisions can occur so the regularized flow is nothing else than the usual flow. The arguments used above for the case of an attractive potential work as well, except that because of (8), $\tilde{u}$ cannot have frequency set above 0 , since this is contained in the elliptic region for $\tilde{p}$. 
Finally the case of many centers can be treated exactly the same way. This completes the proof of the theorem.

\section{References}

[Ea] Easton, R.: Regularization of vector fields by surgery. J. Diff. Eq. 10, 92-99 (1971)

[Le] Lebeau, G.: Régularité Gevrey 3 pour la diffraction. Commun. P.D.E. 9 (1984)

[Gu-St] Guillemin, V., Sternberg, S.: Geometric Asymptotics, A.M.S. Surveys vol. 14 (1977)

[He-Ro] Helffer, B., Robert, D.: Calcul fonctionnel par la transformation de Mellin et opérateurs admissibles. J. Funct. Anal. 53, 246-268 (1983)

[He-Sj] Helffer, B., Sjöstrand, J.: Equation de Schrödinger avec champ magnétique et équation de Harper. Lectures Notes in Physics vol. 345, 118-197. Berlin, Heidelberg, New York: Springer 1989

[Hö] Hörmander, L.: The analysis of linear partial differential operators vols. III and IV. Berlin, Heidelberg, New York: Springer 1985

[Ma] Martinez, A.: Estimations de l'effet tunnel pour le double puits II. Etats hautement excités. Bull. Soc. Math. France 116, 199-229 (1988)

[Mo] Moser, J.: Regularization of Kepler's problem and the averaging method on a manifold. Commun. Pure Appl. Math. 23, 609-636 (1970)

[Ro] Robert, D.: Autour de l'approximation semiclassique. Basell: Birkhaüser 1983

[Sa] Saari, D.: A Visit to the Newtonian $N$-body Problem via Elementary Complex Variables. American Math. Monthly, February 1990, 105-119

[Sj] Sjöstrand, J.: Propagation of analytic singularities for second order Dirichlet problems I and II. Commun. P.D.E. 3, 41-94, 187-207 (1980)

[S-S] Stiefel, E. L., Scheifele, G.: Linear and Regular Celestial Mechanics, Grundlehren der Mathematischen Wissenschaften Band 174. Berlin, Heidelberg, New York: Springer 1975

Communicated by B. Simon 